\title{
QoS Routing in Multihop Packet Radio Environment
}

\author{
Yu-Ching Hsu ${ }^{1} \quad$ Tzu-Chieh Tsai ${ }^{2} \quad$ Ying-Dar Lin ${ }^{1}$
}

\begin{abstract}
In this paper, we present a method to compute the path bandwidth for the DSDV-based routing algorithm. The addressed network does not necessarily have a cellular structure and could have no fixed infrastructure. This network can be either stand-alone, or connected to the wired network. Each mobile station has to relay packets for others, thus achieving multihop routing. To calculate the available bandwidth of a path in this environment, it is incorrect to simply compute the minimum bandwidth of the links along the path. There are two crucial steps in the path bandwidth computation process of this multihop environment: (1) intersecting the sets of common free slots of two adjacent links, and (2) dividing the intersection for the adjacent links to share. We present two bandwidth computation rules including the Half rule and the Floating rule. Numerical results are given to evaluate the performance of applying these rules to the DSDV-based QoS routing algorithm.
\end{abstract}

\section{Introduction}

The recently proposed micro-cellular and pico-cellular architectures for Personal Communication Service(PCS) [1] increase the possibility of introducing multihop wireless routing. Multihop routing can fundamentally reduce the number of base stations and tolerate base station failure. Fig. 1 shows the connection can be recovered by multihop routing when the wired base station B fails.

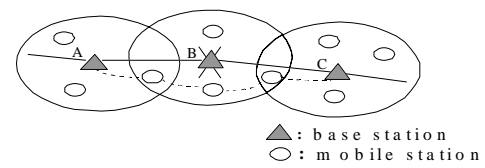

Fig. 1. Cellular base station failure and multihop routing.

Previous works on packet radio networks such as PRNET[2] and the Duct-routing protocol[3] did not consider the support for real-time services and suffered
${ }^{1}$ from the hidden terminal problem[4].

Recently, some new works were conducted for multihop wireless networks [5], [6], and [7]. The routing algorithm in [5] is proposed to avoid the 'false reply' problem in [8]. The hierarchical routing protocol in [6] needs each entity in the architecture to maintain a small fraction of the topology information to help routing in the hierarchical network. However, these two algorithms do not consider bandwidth reservation for the real-time traffic. In the QoS (Quality of Service) study in [7], the routing protocol is based on DSDV[9](DestinationSequenced Distance-Vector) and the bandwidth reservation request is forwarded hop-by-hop along the path to the destination. This algorithm in general does not compute the bandwidth of a path in making its routing decision. Considering the path bandwidth in making the routing decision has the advantage that the call blocking probability can be lowered.

In this paper, we investigate the problem of computing the path bandwidth in order to support QoS routing in the ad-hoc network. We assume the MAC (Medium Access Control) layer to be channelized by the CDMA spread spectrum modulation and each channel is slotted by TDMA. Mobile stations are grouped into clusters by performing the clustering algorithm[10] periodically with lowest-ID scheme[11]. This in effect emulates the cellular structure. Different code sequences are assigned to these clusters so that transmissions in different clusters do not interfere with each other. As shown in Fig. 2, A and C can transmit packets to $\mathrm{B}$ and $\mathrm{D}$, respectively, on the same time slot. We call this CS(Channelized and Slotted)-MAC.

As we shall find in this CS-MAC ad-hoc network, the available bandwidth of a path is not simply the minimum bandwidth of the links along the path. The sets of

Corresponding author: Ying-Dar Lin, email: ydlin@cis.nctu.edu.tw, FAX: +886-3-5721490, Tel: +886-3-5731899.

1. The authors are with Department of Computer and Information Science, National Chiao Tung University, Hsinchu, Taiwan.

2. The author is with Computer Science Department, National Chengchi University, Taipei, Taiwan. 
common free slots of adjacent links should be considered in the computation. In section 2, we discuss the common free slot problem involved in computing the available path bandwidth in the CS-MAC ad-hoc networks. In section 3, we present two rules to divide the overlapped common free slots among channels. Their performance in the DSDV-based bandwidth routing algorithm is described in section 4 . We give conclusions and point out future work in section 5 .

\section{Problem Description}

To provide QoS for connections, we have to compute the available bandwidth of a path. Beyond our initial intuition, bandwidth computation in the CS-MAC ad-hoc networks turns out to be much more complicated than in the wired networks. In Fig. 2, the time slot availability for these four stations is shown on the right-hand side. Assume that a frame cycle has ten time slots and those time slots marked with $\mathrm{F}$ are free, i.e. not reserved for any stations. The hop counts of the paths $\mathrm{CD}, \mathrm{BD}$ and $\mathrm{AD}$ are 1, 2 and 3, respectively. Since stations $C$ and $D$ have three common free slots, $\{1,2,5\}, B W(C D)=3$. Similarly, we can have $\mathrm{BW}(\mathrm{BC})=3$ and $\mathrm{BW}(\mathrm{AB})=2$.

In the wired environment, $\mathrm{BW}(\mathrm{BD})=3$ can be implied immediately by $\mathrm{BW}(\mathrm{BD})=\operatorname{Min}(\mathrm{BW}(\mathrm{BC}), \mathrm{BW}(\mathrm{CD}))$. However, BW(BD) should be 2 in this example of ad-hoc networks. It is mainly because the intersection of common free slots of $\mathrm{B}$ and $\mathrm{C},\{1,2,3\}$, and common free slots of $\mathrm{C}$ and $\mathrm{D},\{1,2,5\}$, is a non-empty set, $\{1,2\}$. Since station $\mathrm{C}$ can not receive and transmit packets on the same time slot, the set $\{1,2\}$ has to be shared among link BC and link CD. Suppose $\{1,2\}$ is divided into $\{1\}$ for link $B C$ and $\{2\}$ for link $C D$, now station $C$ can receive from $B$ on $\{1,3\}$ and transmit to $\mathrm{D}$ on $\{2,5\}$. Thus, we have $\mathrm{BW}(\mathrm{BD})=2$. There are two problems involved in this path bandwidth computation process. The first problem is how station B knows the set of common free slots of two adjacent links, and the second problem is how to share these overlapped common free slots. To solve these problems, the stations have to exchange some messages with each other. Another problem is the hidden terminal problem[4] which also needs to be considered in the ad-hoc network.

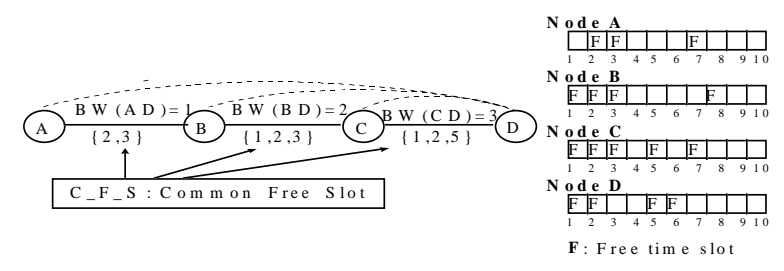

Fig. 2. Common free slots and path bandwidth.

\section{Solution}

After clustering the stations in the system, CDMA and TDMA are applied to channelize the spectrum and slot the channels, respectively. Assume half-duplex operation. Every station builds the bandwidth routing table based on the DSDV-based routing algorithm which is driven by both the path bandwidth and path delay.

\section{A. Clustering}

To take advantage of frequency reuse, we emulate the cellular structure by performing the clustering algorithm periodically [10] with the lowest-ID scheme[11]. A station could be a cluster head, a gateway, or just an usual station. Once a station is held to be a cluster head, all its neighbors belong to the same cluster. A station belonging to two clusters is to play the role of gateway. CDMA mechanism is used to partition the clusters by assigning different code sequences to clusters, and TDMA is enforced within a cluster. Synchronization is done on a per-cluster basis in order to run TDMA.

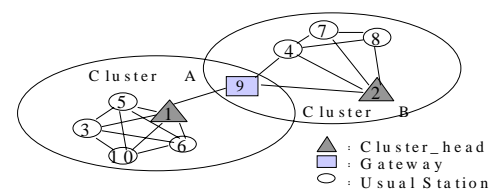

Fig. 3. Clustered multihop wireless network: emulating the cellular structure.

Fig. 3 shows the result of clustering according to station's position and identifier. Station 1 and 2 are the cluster heads of Cluster A and B respectively, and station 9 is a gateway.

\section{B. Bandwidth computation rules}

To solve the hidden terminal problem, all stations refer to the reservation status of the cluster head's time slots while building their routing tables. Since the cluster head can hear all the other stations in its cluster, it has the most complete information of reservation status.

The routing table is refreshed fast enough to clear up the obsolete information due to topology change. To build this table, every station periodically transmits a "free_slot" message which contains its slot reservation status. As shown in Fig. 2, station C can figure out the set of common free slots on link CD, $\{1,2,5\}$, when it receives the message, free_slot $(1,2,5,6)$, from station $D$, and vice versa. Denote the set of common free slots of a link $\mathrm{AB}$ as $\mathrm{CFS}(\mathrm{AB})$.

The propagation path in Fig. 4, derived from Fig. 2, 
shows how stations $\mathrm{C}, \mathrm{B}$ and A obtain the bandwidth information for the route to station D. Station C broadcasts a "bw" message containing $\{1,2,5\}$, the CFS(CD), such that station $B$ can compute the available bandwidth of path BCD by first intersecting $\mathrm{CFS}(\mathrm{BC})$, $\{1,2,3\}$, with $\{1,2,5\}$ in the "bw" message. Then station $\mathrm{B}$ decides that it can divide the intersection $\{1,2\}$ into $\{2\}$ and $\{1\}$ so that it can use slots $\{2,3\}$ on link BC, and leaves slots $\{1,5\}$ for station $C$ to forward packets on link CD. Again station B broadcasts a "bw" message containing $\{2,3\}, \operatorname{FSP}(\mathrm{BD})$, so that station A may use $\{3\}$ to send packets to station $\mathrm{D}$, while leaving $\{2\}$ for station $\mathrm{B}$ to forward on link BC. Note that FSP(BD) denotes the set of free slots along the path connecting $\mathrm{B}$ and $\mathrm{D}$. If there are multiple paths between $\mathrm{B}$ and $\mathrm{D}$, the one with the shortest path is selected. If there is a tie, the one with the maximum bandwidth is selected.

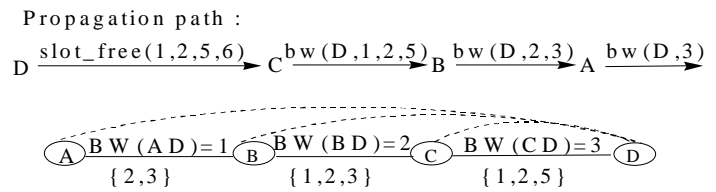

Fig. 4. Propagation path for station A, B and C to compute bandwidth to station $\mathrm{D}$.

If the hop count of a path is one, it is for sure that the bandwidth of the path is the number of elements in CFS on the link. What if the hop count is greater than one, as shown in Fig. 5? According to the above example, station A has to know $\operatorname{CFS}(\mathrm{AB})$ and $\operatorname{FSP}(\mathrm{BC})$.

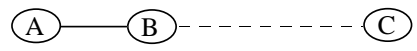

Fig. 5. A path from station A to station $\mathrm{C}$.

Fig. 6 shows the relationship between $\operatorname{CFS}(\mathrm{AB})$ and $\mathrm{FSP}(\mathrm{BC})$, i.e. $\mathrm{X} \cup \mathrm{Z}$ and $\mathrm{Z} \cup \mathrm{Y}$, where $\mathrm{Z}$ is the intersection of CFS(AB) and FSP(BC). Station A needs to divide these two sets into three groups, $\mathrm{X}, \mathrm{Y}$ and $\mathrm{Z}$, when it computes bandwidth. At station A, X, Y and $\mathrm{Z}$ can be computed, in this order, as

$$
\begin{array}{ll}
Z=C F S(A B) \cap F S P(B C) & \text { and } \quad z=\operatorname{Num}(Z), \\
X=C F S(A B)-Z & \text { and } \quad x=\operatorname{Num}(X), \\
Y=F S P(B C)-Z & \text { and } y=\operatorname{Num}(Y),
\end{array}
$$

where Num gives the number of elements in a set, CFS is the set of common free slots of a link, and FSP is the set of available free slots along a path.

Note that each free slot in $\mathrm{Z}$ can be used to carry a packet on link $\mathrm{AB}$ or on path $\mathrm{BC}$, but not both. Thus, how to allocate $\mathrm{Z}$ to link $\mathrm{AB}$ and path $\mathrm{BC}$ is an important issue. We have developed two bandwidth computation rules, the Half rule and the Floating rule, which are based on the relationship between the sizes of these three groups. They are designed to solve the issue of allocating $\mathrm{Z}$ to link $\mathrm{AB}$ and path BC. In section 4, we will find these two rules result in a tradeoff between loss, blocking, and dropping rates.

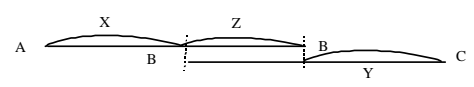

Fig. 6. Three slot groups of a link and the adjacent path.

\section{Half rule}

The basic idea of the Half rule is to let link $A B$ and path $\mathrm{BC}$ share the overlapped group $\mathrm{Z}$ equally. Hence station $B$ can use half of the $Z$ group and the whole $X$ group to receive packets from station $A$ and forwards packets to station $\mathrm{C}$ in the other half of the $\mathrm{Z}$ group and the whole $\mathrm{Y}$ group.

We formulize the Half rule into the following procedure, where $\mathrm{X}, \mathrm{Y}$ and $\mathrm{Z}$ are defined as above, and the relationship between stations $\mathrm{A}, \mathrm{B}$ and $\mathrm{C}$ is shown in Fig. 5:

Procedure $\mathrm{H}$ :

If distance $(\mathrm{AC})=1, \mathrm{BW}(\mathrm{AC}) \leftarrow \operatorname{Num}(\mathrm{CFS}(\mathrm{AC}))$; otherwise, $\mathrm{BW}(\mathrm{AC}) \leftarrow \operatorname{Min}((\mathrm{x}+\mathrm{z} / 2),(\mathrm{y}+\mathrm{z} / 2))$,

where distance $(\mathrm{AC})$ is the hop count of the path.

\section{Floating rule}

The basic idea of the Floating rule is to let the cutting point float on the overlapped group $\mathrm{Z}$, and the location of cutting point depends on the sizes of groups $\mathrm{X}, \mathrm{Y}$, and $\mathrm{Z}$. We define the Floating rule procedure as follows, where the relationship between stations $\mathrm{A}, \mathrm{B}$ and $\mathrm{C}$ is again in Fig. 5:

$$
\begin{aligned}
& \text { Procedure F: } \\
& \text { If distance }(\mathrm{AC})=1, \mathrm{BW}(\mathrm{AC}) \leftarrow \operatorname{Num}(\mathrm{CFS}(\mathrm{AC})) \text {; } \\
& \text { otherwise, 1. If }(x+y+z) / 2<x \text {, } \\
& \mathrm{BW}(\mathrm{AC}) \leftarrow \mathrm{y}+\mathrm{z}, \\
& \text { 2. If }(x+y+z) / 2>x+z \text {, } \\
& \mathrm{BW}(\mathrm{AC}) \leftarrow \mathrm{x}+\mathrm{z} \text {, } \\
& \text { 3. If } x<(x+y+z) / 2<x+z \text {, } \\
& \mathrm{BW}(\mathrm{AC}) \leftarrow(\mathrm{x}+\mathrm{y}+\mathrm{z}) / 2 \text {. }
\end{aligned}
$$

\section{Simulation Study}

\section{A. Simulation Model}

The simulated environment consists of 20 mobile stations moving in constant speed within a rectangular area of $150 * 600 \mathrm{~m}^{2}$. The propagation distance of the transmitted packets is 150 meters. We neglect the overhead of control phase in a TDMA frame cycle, and assume the length of transmit phase of a cycle to be 100 $\mathrm{ms}$. The transmit phase is divided into 10 time slots, each of which is $10 \mathrm{~ms}$. In the simulation runs, 20 stations move at the same speed, $11 \mathrm{~km}$ per hour.

The routing table is refreshed every frame cycle, i.e. 
0.1 second. The greatest distance of a station may move before the routing table is updated is 0.31 meter, i.e. $11 \mathrm{~km} / 3600 \mathrm{sec} \times 0.1 \mathrm{sec}$, which is relative small as compared to 150 meters, the propagation distance of transmitted packets. This shows that the frequency of routing table update is high enough to timely reflect the current topology.

The interarrival time and the service time of calls are both exponentially distributed. The mean interarrival time is 11 seconds, and the mean service time is 90 seconds. The bandwidth required for a call can be 1 or 2 time slots depending on the voice connection quality. Assume that the radio transmission capacity is $1 \mathrm{Mbps}$, and the synchronization overhead is $5 \mathrm{~ms}$ for each $10 \mathrm{~ms}$ time slot. Thus, if a call requests one time slot, it is equivalent to $1 \mathrm{Mbps} \times 5 \mathrm{~ms} / \mathrm{slot} \times 1$ slot/frame $\times$ $10 \mathrm{frame} / \mathrm{sec}=50 \mathrm{kbps}$.

\section{B. Numerical Results}

In this multihop environment, the number of channels may change due to station mobility, which makes it difficult to measure the total network capacity and channel throughput, we define channel offered load as $\frac{\text { Num (Offered_Packet) }}{\operatorname{Num}(\text { Slot }) * N u m \text { (avg (Channel)) }}$, and collect the packet loss rate, call blocking rate and call dropping rate instead of channel throughput. A lost packet will not necessarily be retransmitted for real-time traffic. When bandwidth is insufficient, a call will be blocked as a new call arrives or be dropped when it needs to change path due to topological changes.

\section{Benefits of QoS control}

To see the effect of bandwidth computation we compare three different operation models: QoS without re-routing, QoS with re-routing and NoQoS. The Half rule is applied to both of the first two models, and no bandwidth computation rule is applied in the third model. The first model is designed to measure the effect of station mobility where a call is dropped directly when the original path is broken.

We attribute packet loss due to mobility, because packets may be dropped before the call be switched to a new path. From Fig. 7, we can see the model of NoQoS, without bandwidth computation, results in serious loss.

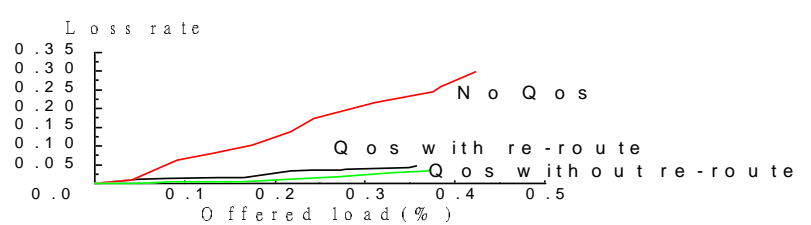

Fig. 7. Offered Load v.s Loss Rate.

In the model of NoQoS, a station would accept the call request as long as it can find enough free slots from its local bandwidth reservation table, thus it has much lower call blocking rate than the other two models as shown in Fig. 8 .

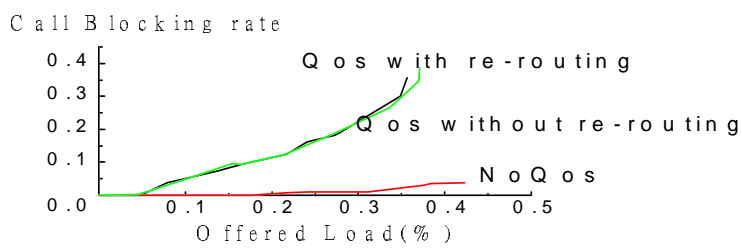

Fig. 8. Offered Load vs. Call Blocking Rate.

As shown in Fig. 9, when offered load is high, it is not easy for the connection source to find a new proper path and thus the call is likely to be dropped directly. We can also see that the model of QoS without re-routing cannot adjust to mobility because of its definition. The model of NoQoS has very low dropping rate because the station only considers its own bandwidth reservation status. However, the bandwidth may not be available, resulting in serious packet loss.

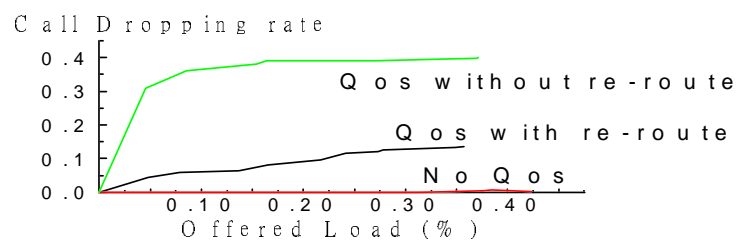

Fig. 9. Offered Load vs. Call Dropping Rate.

\section{Half rule vs. Floating rule}

From Fig. 10, 11 and 12 we can see that the Floating rule performs slightly better than the Half rule, since the computed bandwidth is greater than the one by the other rule. Once a call is accepted in the model adopting the Floating rule, it is easier to find a new path in the rerouting process, which results in the lower call dropping rate. However, a new call will be blocked if the available bandwidth is less than one or two units. In this case, the difference of these two rules would be small and thus leads to about the same call blocking rate.

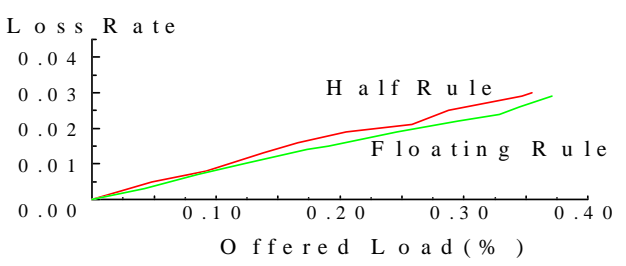

Fig. 10. Offered Load vs. Loss Rate. 


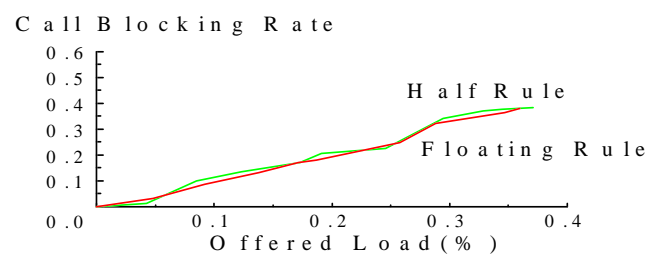

Fig. 11. Offered Load vs. Call Blocking Rate.

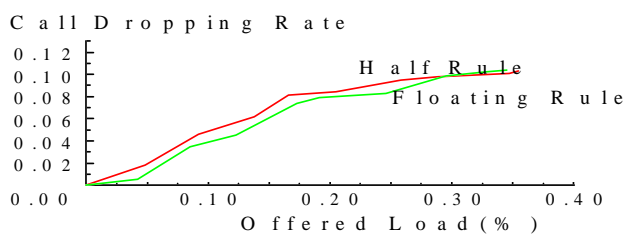

Fig. 12. Offered Load vs. Call Dropping Rate.

\section{Conclusions and Future Work}

In this paper, the CS-MAC is assumed. To assist the DSDV-based routing algorithm in selecting and reserving the path with the required bandwidth, techniques in computing the bandwidth of a path are developed. Unlike the computation in the wired network where minimum bandwidth of the links along the path is taken, the accurate computation here needs to consider the intersection of the common free slots of two adjacent links. This overlapped set of time slots needs to be divided and shared by the adjacent links. Rules on how to divide it are also presented.

The simulation results show the benefits of bandwidth computation and reservation where packet loss rate can be lowered and call dropping rate is controlled. It helps to advance the QoS routing for real time traffic in the ad-hoc network. We can also see that the Floating rule performs slightly better than the Half rule.

Since packets may be lost and the call could be dropped in the re-routing process, how reliable a path is should be further considered in choosing the path. We intuitively define a weighted rule based on the power strength of the received signal as follows:

$\mathrm{BW}^{\mathrm{w}}(\mathrm{AB})=\mathrm{w} \times \mathrm{BW}(\mathrm{AB})$,

where $\mathrm{w}=0$ if $\mathrm{P}_{\mathrm{B}}<\mathrm{P}$ and $\triangle \mathrm{P}_{\mathrm{B}}<0$,

(i.e. $\mathrm{B}$ is leaving and its signal is weak), $\mathrm{w}=\triangle \mathrm{P}_{\mathrm{B}} / \mathrm{P}$ if $\mathrm{P}_{\mathrm{B}}<\mathrm{P}$ and $\triangle \mathrm{P}_{\mathrm{B}}>0$, (i.e. $\mathrm{B}$ 's signal is weak but $\mathrm{B}$ is getting closer), $\mathrm{w}=1+\triangle \mathrm{P}_{\mathrm{B}} / \mathrm{P}$ if $\mathrm{P}_{\mathrm{B}}>\mathrm{P}$ and $\triangle \mathrm{P}_{\mathrm{B}}<0$, (i.e. $\mathrm{B}$ 's signal is strong but $\mathrm{B}$ is leaving), $\mathrm{w}=1$ if $\mathrm{P}_{\mathrm{B}}>\mathrm{P}$ and $\angle \mathrm{P}_{\mathrm{B}}>0$

closer).

(i.e. $\mathrm{B}$ 's signal is strong and $\mathrm{B}$ is getting even

Note that $\mathrm{P}$ is a constant threshold, $\mathrm{P}_{\mathrm{B}}$ is the power strength of the received signal from station $\mathrm{B}, \triangle \mathrm{P}_{\mathrm{B}}$ is the change of strength of the received signal from station $\mathrm{B}$, and $\mathrm{BW}(\mathrm{AB})$ is the bandwidth of the link computed either by the Half rule or by the Floating rule. If the hop count between station A and B is greater than one, the overall weight of a path should be the product of weights of all links along the path.

\section{References}

[1] Luis M. Correia and Ramjee Prasad, "An Overview of Wireless Broadband Communications", IEEE Communications, Vol. 35, No.1, pp. 28-33, Jan 1997.

[2] Robert E. K., Steven A. G., Jerry B., and Ronald C. K., "Advances in Packet Radio Technology", Proceedings of the IEEE, Vol. 66, pp.1468-1496, 1978.

[3] Nachum Shacham, Earl J. Craighill, and Andrew A. Poggio, "Speech Transport in Packet-Radio Networks with Mobile Stations", IEEE Journal of Selected Areas in Communications, Vol. SAC-1, No.6, pp. 1084-1097, Dec 1983.

[4] F. A. Tobagi and L. Kleinrock, "Packet Switching in Radio Channels, Part II: The Hidden-Terminal Problem in Carrier Sense Multiple Access and the Bus-Tone Solution", IEEE Trans. Commun., COM23, pp.1417-1433, 1975,.

[5] Vincent D. Park, and M. Scott Corson, "A Highly Adaptive Distributed Routing Algorithm for Mobile Wireless Networks", IEEE INFOCOM'97, Kobe, Japan, April 1997.

[6] Ian F. Akyildiz, Wei Yen, and Bulent Yener, "A New Hierarchical Routing Protocol for Dynamic Multihop Wireless Networks", IEEE INFOCOM'97, Kobe, Japan, 1997.

[7] Chunhung Richard Lin, and Mario Gerla, "Asynchronous Multimedia Multihop Wireless Networks”, IEEE INFOCOM'97, Kobe, Japan, April 1997.

[8] M. S. Corson and A. Ephremides, "A Distributed Routing Algorithm for Mobile Wireless Networks", ACM Wireless Networks, pp. 61-81, 1995.

[9] C. E. Perkins, and P. Bhagwat, "Highly Dynamic Destination-Sequenced Distance-Vector Routing (DSDV) for Mobile Computers", ACM SIGCOMM'94, pp.234-244, 1994.

[10] Mario Gerla, and Jack Tzu-Chieh Tsai, "Multicluster Mobile Multimedia Network", ACM-Baltzer Journal of Wireless Networks, Vol.1, No.3, pp.255-265, 1995.

[11] A. Ephremides, J.E. Wieselthier and D.J. Baker, "A Design Concept for Reliable Mobile Radio Networks With Frequency Hopping Signaling”, Proceedings of the IEEE, Vol. 75, pp. 56-73, 1987. 